\title{
An Annular Plate Model in Arbitrary Lagrangian-Eulerian Description for the DLR FlexibleBodies Library
}

\author{
Andreas Heckmann*, Stefan Hartweg* and Ingo Kaiser* \\ ${ }^{*}$ German Aerospace Center (DLR), Institute of Robotics and Mechatronics \\ Oberpfaffenhofen, 82234 Wessling, Germany
}

\begin{abstract}
The bending deformation of rotating annular plates and the associated vibration behaviour is important in engineering applications which range from automotive or railway brake systems to discs that form essential components in turbomachinery.

In order to extend the capabilities of the DLR FlexibleBodies library for such use cases, a new Modelica class has been implemented which is based on the analytical description of an annular Kirchhoff plate. In addition the so-called Arbitray Langrangian-Eulerian (ALE) representation has been adopted so that rotating and non-rotating external loads may be applied conventiently to rotating plates.

Besides these particularities the new class AnnularPlate completely corresponds to the concept of FlexibleBodies library with the two already available model classes Beam and ModalBody.

This paper gives an overview on the theoretical background of the new class AnnularPlate, explains the usage and presents application examples.

Keywords: Arbitrary Lagrangian-Eulerian approach, annular Kirchhoff plate, flexible multibody system
\end{abstract}

\section{Introduction}

The commercial DLR FlexibleBodies library presented in 2006 [1] contains two different types of model classes: The Beam model employs an analytical description of the deformation field, while a general ModalBody model is defined in such a way that the dynamic behaviour of a body with an arbitrary geometrical shape can be simulated if suitable finite element data of the body exist.

The new model class AnnularPlate introduced in this paper is implemented in the same manner as it applies for the Beam model. The analytical description of an annular Kirchhoff plate has been used to define the object-oriented data structure called "Standard Input Data of flexible bodies" (SID), see [2], which is the general base of all models in the DLR FlexibleBodies library.

Rotating discs are a very common structure type in mechanical engineering. But their modeling often has to cope with the difficulty to describe non-rotating forces acting on the disc such as the normal and friction forces at a disc brake. Usually this requires a contact formulation in order to evaluate which material point of the disc is in contact with the pad at the considered point in time.

Due to the so-called Arbitrary LagrangianEulerian description it is possible to provide a standard Modelica multibody frame connector which is however not linked to a material point of the plate, but slides over the surface of the plate as it is given for the brake disc-pad contact point. No contact problem has to be formulated and solved and normal and friction forces are convenient to apply at this frame connector.

In addition the AnnularPlate model is capable of defining material-fixed points on the plate with frame connectors to which forces, other bodies such as unbalances, springs etc. may be attached in the usual way.

\section{Theoretical Background}

This section shortly summarizes well-known fundamentals on structural dynamics of annular plates and on multibody dynamics. The modeling approach of the FlexibleBodies library utilizes these fundamentals and will be introduced in Sec. 3 .

\subsection{Partial Differential Equation}

The partial differential equation (PDE) of a freely vibrating, homogeneous Kirchoff plate with transverse deformation $w$ in the mid-plane of the plate as a function of the radius $r$, the angle $\phi$ and time $t$, i.e. 
$w=w(r, \phi, t)$, reads

$$
D \Delta \Delta w+\hat{\rho} \ddot{w}=0,
$$

where $\Delta$ denotes the Laplace operator and $\hat{\rho}$ represents the mass per unit area $[3,(1.1)] . D$ is the bending rigidity of the plate according to

$$
D:=\frac{E h^{3}}{12\left(1-v^{2}\right)}
$$

and depends on the Young's modulus $E$, the plate thickness $h$ and the Poisson number $v$.

An analytical solution to (1) is assumed in the form

$$
w(r, \phi, t)=R(r) \cdot \Phi(\phi) \cdot q(t),
$$

so that the PDE (1) can be separated in three ordinary differential equations (ODE) for $R(r), \Phi(\phi)$ and $q(t)$, respectively [4, 4.3.15].

For $R(r)$ the Bessel-type ODE

$$
\begin{aligned}
r^{4} R^{\prime \prime \prime \prime} & +2 r^{3} R^{\prime \prime \prime}-\left(1+2 k^{2}\right)\left(r^{2} R^{\prime \prime}-r R^{\prime}\right)+ \\
& +\left(k^{4}-4 k^{2}-\lambda^{4} r^{4}\right) R=0,()^{\prime}:=\frac{\mathrm{d}}{\mathrm{d} r},
\end{aligned}
$$

is obtained [5, (5.1-120)]. The parameter $k$ relates (4) to $\Phi(\phi)$ in (3) and represents the wavenumber or the number of nodal diameters in Fig. 2. The parameter $\lambda$ depends on the eigenvalue $\omega$ of the ODE for $q(t)$ :

$$
\lambda^{2}:=\omega \sqrt{\frac{\hat{\rho}}{D}} .
$$

Bessel and modified Bessel functions of first and second kind satisfy (4) and have to be selected in such a way that the boundary conditions at the inner and the outer radius of the annular plate are considered.

Harmonic waves provide a solution with respect to the angular coordinate, i.e. $\Phi(\phi)=\cos \left(k \phi+\psi_{k}\right)$ with offset angle $\psi_{k}$ and $\Phi(\phi)=\Phi(\phi+2 \pi)$.

Finally, the time-dependency of the displacements $q(t)$ are as well assumed to be harmonic, e.g. $q(t)=\sin (\omega t)$.

Note that (1) has an infinite number of solutions, out of which only a reduced, finite number of eigenvalues $\omega$ and associated deformation fields, the eigenforms in Fig. 2, are considered for numerical analysis.

\subsection{Multibody Framework}

The mechanical description in multibody dynamics is based on the floating frame of reference approach, i.e. the absolute position $\boldsymbol{r}=\boldsymbol{r}(\boldsymbol{c}, t)$ of a specific body particle is subdivided into three parts: the position vector
$\boldsymbol{r}_{R}=\boldsymbol{r}_{R}(t)$ to the body's reference frame, the initial position of the body particle within the body's reference frame, i.e. the Lagrange coordinate $c \neq c(t)$, and the elastic displacement $\boldsymbol{u}(\boldsymbol{c}, t)$ :

$$
\boldsymbol{r}=\boldsymbol{r}_{R}+\boldsymbol{c}+\boldsymbol{u}
$$

where all terms are resolved w.r.t. the body's floating frame of reference $(R)$.

Therefore the angular velocity of the reference frame $\boldsymbol{\omega}_{R}$ have to be taken in account when the kinematic quantities velocity $\boldsymbol{v}$ and acceleration $\boldsymbol{a}$ of a particle are derived:

$$
\begin{aligned}
& \boldsymbol{v}=\tilde{\boldsymbol{\omega}}_{R} \boldsymbol{r}+\dot{\boldsymbol{r}}=\boldsymbol{v}_{R}+\tilde{\boldsymbol{\omega}}_{R}(\boldsymbol{c}+\boldsymbol{u})+\dot{\boldsymbol{u}}, \\
& \boldsymbol{a}=\boldsymbol{a}_{R}+\left(\dot{\tilde{\boldsymbol{\omega}}}_{R}+\tilde{\boldsymbol{\omega}}_{R} \tilde{\boldsymbol{\omega}}_{R}\right)(\boldsymbol{c}+\boldsymbol{u})+2 \tilde{\boldsymbol{\omega}}_{R} \dot{\boldsymbol{u}}+\ddot{\boldsymbol{u}},
\end{aligned}
$$

where the $(\tilde{)})$-operator is used to replace the vector cross product by a multplication with an appropriate skew-symmetric matrix, so that e.g. the identity $\boldsymbol{\omega} \times$ $\boldsymbol{c}=\tilde{\boldsymbol{\omega}} \boldsymbol{c}$ holds.

The decomposition in (6) makes it possible to superimpose a large non-linear overall motion of the reference frame with small elastic deformations.

The displacement field of the annular plate is approximated by a first order Taylor expansion with space-dependent mode shapes $\boldsymbol{\Phi}(\boldsymbol{c}) \in \mathbb{R}^{3, n}$ and timedependent modal amplitudes $\boldsymbol{q}(t) \in \mathbb{R}^{n}[2]$ :

$$
\boldsymbol{u}=\boldsymbol{\Phi} \boldsymbol{q}
$$

Note that the description of the annular plate is limited to this first order expansion in this initial implementation, so that plate buckling phenomena are not covered, see [6], [7, Ch. 1]. The second order displacement field of an annular plate currently is a field of research at the DLR.

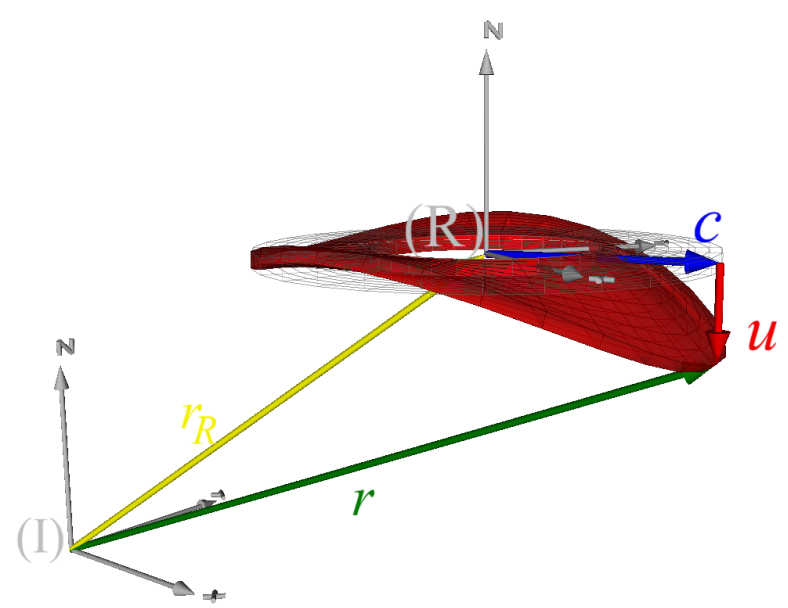

Figure 1: Vector chain to specify the position $\boldsymbol{r}$ resolved in the floating frame of reference $(\mathrm{R})$. 
The kinematic quantities together with the vector of applied forces $\boldsymbol{f}_{e}$ are inserted into Jourdain's principle of virtual power:

$$
\delta \boldsymbol{v}^{T} \int_{\text {body }}\left(\mathrm{d} \boldsymbol{f}_{e}-\boldsymbol{a} \mathrm{d} m\right)=0 .
$$

Subsequently, the equations of motion of an unconstrained flexible body are formulated neglecting deflection terms of higher than first order [2, (38)]:

$$
\begin{aligned}
\left(\begin{array}{ccc}
m \boldsymbol{I}_{3} & & \text { sym. } \\
m \tilde{\boldsymbol{d}}_{C M} & \boldsymbol{J} & \\
\boldsymbol{C}_{t} & \boldsymbol{C}_{r} & \boldsymbol{M}_{e}
\end{array}\right)\left[\begin{array}{c}
\boldsymbol{a}_{R} \\
\dot{\boldsymbol{\omega}}_{R} \\
\ddot{\boldsymbol{q}}
\end{array}\right]= \\
=\boldsymbol{h}_{\omega}-\left[\begin{array}{c}
\mathbf{0} \\
\mathbf{0} \\
\boldsymbol{K}_{e} \boldsymbol{q}+\boldsymbol{D}_{e} \dot{\boldsymbol{q}}
\end{array}\right]+\boldsymbol{h}_{e},
\end{aligned}
$$

where the following quantities and symbols appear: $m \quad$ body mass

$I_{3} \quad 3 \times 3$ identity matrix

$\boldsymbol{d}_{C M}(\boldsymbol{q}) \quad$ position of center of mass

$\boldsymbol{J}(\boldsymbol{q}) \quad$ inertia tensor

$\boldsymbol{C}_{t}(\boldsymbol{q}) \quad$ inertia coupling matrix

$\boldsymbol{C}_{r}(\boldsymbol{q}) \quad$ inertia coupling matrix

$\boldsymbol{h}_{\omega}(\boldsymbol{\omega}, \boldsymbol{q}, \dot{\boldsymbol{q}}) \quad$ gyroscopic and centripetal forces

$\boldsymbol{h}_{e}$

$\boldsymbol{M}_{e} \quad$ structural mass matrix

$\boldsymbol{K}_{e} \quad$ structural stiffness matrix

$\boldsymbol{D}_{e} \quad$ structural damping matrix

If, for the sake of demonstration, the body is assumed to be rigid, those rows and columns in (11) vanish that are associated with the generalised deformational acceleration $\ddot{\boldsymbol{q}}$. Since (11) is formulated in terms of the translational and angular acceleration of the floating frame of reference, such reduction leads to the classical Newton-Euler equations of a rigid body. Therefore, SHABANA calls (11) the generalised Newton-Euler equations of an unconstrained deformable body in [8, Sec. 5.5].

On the other hand, if the motion of the reference frame is constrained to be zero, (11) is reduced to the classical structural equation

$$
\boldsymbol{M}_{e} \ddot{\boldsymbol{q}}+\boldsymbol{D}_{e} \dot{\boldsymbol{q}}+\boldsymbol{K}_{e} \boldsymbol{q}=\boldsymbol{f}_{e},
$$

where $\boldsymbol{f}_{e}$ is that part of $\boldsymbol{h}_{e}$ that is associated to the rows of $\ddot{\boldsymbol{q}}$.

\section{The Annular Plate Model}

\subsection{Mode Shapes}

In order to specify the spatial shape functions in (9) the knowledge on the analytical solution in (3) is exploited and the displacements are formulated as function of cylindrical coordinates, i.e. $\boldsymbol{\Phi}=\boldsymbol{\Phi}(r, \phi, z), w_{, r}$ and $w_{, \phi}$ are partial derivatives with respect to $r$ or $\phi$ :

$$
\begin{gathered}
\sum_{i=1}^{n} \boldsymbol{\Phi}_{i} q_{i}(t)=\left[\begin{array}{c}
-z\left(\cos (\phi) w_{, r}-\frac{\sin (\phi)}{r} w_{, \phi}\right) \\
-z\left(\sin (\phi) w_{, r}+\frac{\cos (\phi)}{r} w_{, \phi}\right) \\
w
\end{array}\right], \\
w=\sum_{l=0}^{l_{m}} \sum_{k=0}^{k_{m}} R_{l}(r) \cdot \cos (k \phi) \cdot q_{i}(t)+\ldots \\
\ldots+\sum_{l=0}^{l_{m}} \sum_{k=1}^{k_{m}} R_{l}(r) \cdot \sin (k \phi) \cdot q_{i}(t), \\
\text { with } i=1,2, \ldots, n, n=\left(l_{m}+1\right)\left(2 k_{m}+1\right) .
\end{gathered}
$$

Since the parameter $k$ may be interpreted as the number of nodal diameters and $l$ as the number of nodal circles, each specific couple $\langle l, k\rangle$ may be visualized by a nodal pattern shown in Fig. 2, which characterizes the shape function or eigenform, respectively.

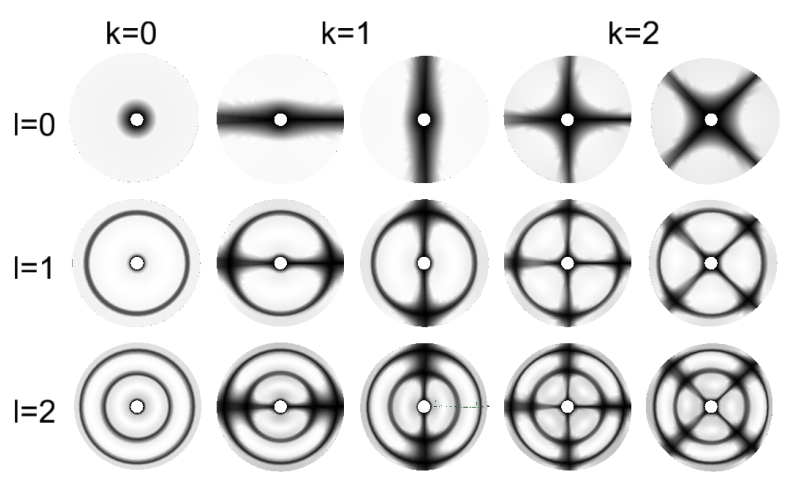

Figure 2: Example nodal diameters $k$ and circles $l$ that characterize annular plate eigenforms. Supported boundary conditions are applied at the inner radius.

For the sake of demonstration Fig. 3 illustrates one exemplary mode shape from Fig. 2 in a different way.

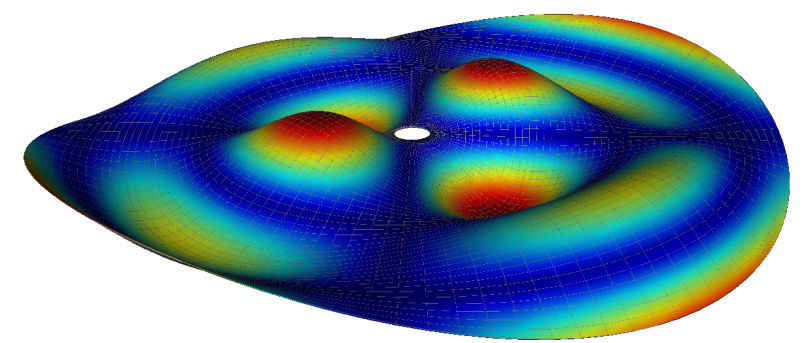

Figure 3: The $k=2$ and $l=2$ mode shape from Fig. 2 in more details, cp. [5, 5.1-29]. 
The number of considered mode shapes in the model is controlled by the values $l_{m}$ and $k_{m}$, which are to be specified by user input parameters of the AnnularPlate class.

The functions $R_{l}(r)$ in (13) correspond to the Bessel functions mentioned in Sec. 2.1. However for the sake of simplicity and robustness of the implementation the original Bessel functions have not been used but a two-step approach is applied. At first the displacement field in radial direction and the underlying ODE (4) is discretized with cubic B-splines $\overline{\boldsymbol{R}}(r)=\left(\bar{R}_{1}(r), \bar{R}_{2}(r), \ldots, \bar{R}_{p}(r)\right)^{T}$ as shown in Fig. 4 taking the boundary conditions at the inner and outer radius into account [9].

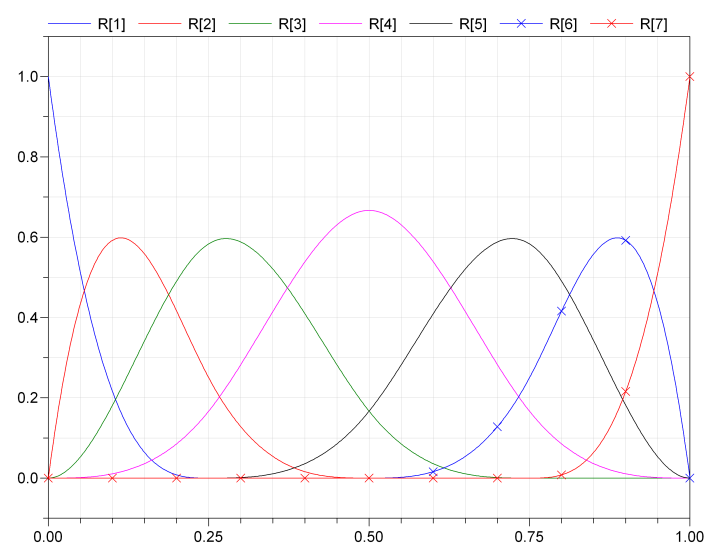

Figure 4: Example set of cubic B-splines for free boundary conditions to initially discretize the displacement field in radial direction.

That way the associated mass $\overline{\boldsymbol{M}}_{e}$ and stiffness matrix $\overline{\boldsymbol{K}}_{e}$ are evaluated. In the second step the problem

$$
\left[\overline{\boldsymbol{M}}_{e} \omega_{n}^{2}+\overline{\boldsymbol{K}}_{e}\right] \boldsymbol{v}_{n}=0
$$

is solved for a specified number of eigenvalues $\omega_{n}$. One specific eigenvector $\boldsymbol{v}_{n=l}$ may then be interpreted to define a fixed linear combination of the initial Bsplines functions in such a way that the associated solution of (4) is approximated, i.e. $R_{l} \approx \boldsymbol{v}_{l}^{T} \cdot \overline{\boldsymbol{R}}$. The accuracy of the approximation may be controlled by the number of the initially used B-splines $p$ in relation to the specified value $l_{m}$. The final result corresponds to the approach in (13).

\subsection{Arbitrary Lagrangian-Eulerian Descrip- tion}

It is now considered that the annular plate performs a in general large rotation around its central axis specified by the angle $\chi(t)$. So far the motion of material

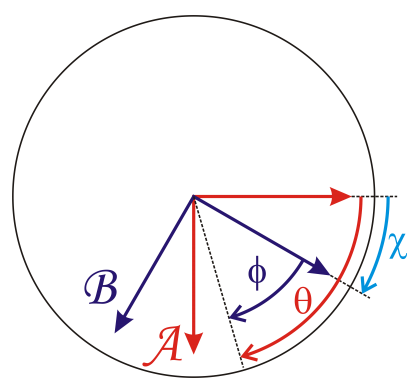

Figure 5: Coordinate transformation with angle $\chi$, that leads from the Langrangian to the ALE-decription.

particles is described in the so-called Lagrangian point of view [10, Sec. I.3], i.e. the floating frame of reference follows the rotation as it is shown for the coordinate system named $\mathcal{B}$ in Fig. 5.

However for specific use cases it may make sense to resolve the deformation of the plate in frame $\mathcal{A}$ in Fig. 5 that follows the complete reference motion of the plate except of the motion expressed by the angle $\chi$. In other words, the observer does not rotate with the plate but looks on the plate from the outside, from a point in rest concerning the rotation with angle $\chi(t)$.

This idea is influenced by the Eulerian description [10, Sec. I.4] widely used in fluid dynamics, where the motion state of the fluid at a fixed point in space is presented. However the concept introduced above combines aspects of the Lagrangian and the Eulerian approach and is therefore known as Arbitrary Lagrangian-Eulerian (ALE) description in literature, see e.g. [11]. Due to the rotational symmetry properties of the annular plate the ALE-description can here be formulated in an elegant way.

For theoretical derivation the coordinate transformation

$$
\phi=\theta-\chi
$$

is defined, where $\theta$ specifies the angular position of an observed point on the annular plate resolved with respect to the ALE-reference system $\mathcal{A}$ in Fig. 5.

Furthermore it is assumed that for every mode shape in (13) that employs a $\sin (k \phi)$-term an associated mode shape is present where the sinus- is replaced by the cosinus-function only, but $R_{l}(r)$ and $k$ are identical, so that mode shape couples $c_{1}$ and $c_{2}$ exist:

$$
\begin{aligned}
& c_{1}(r, \phi)=R_{l}(r) \cdot \sin (k \phi), \\
& c_{2}(r, \phi)=R_{l}(r) \cdot \cos (k \phi) .
\end{aligned}
$$

If the following identities

$$
\begin{aligned}
\sin (k \phi) & =\sin (k \theta) \cos (k \chi)-\cos (k \theta) \sin (k \chi), \\
\cos (k \phi) & =\cos (k \theta) \cos (k \chi)+\sin (k \theta) \sin (k \chi)
\end{aligned}
$$


are inserted into (16), an associated mode couple $\bar{c}_{1}(r, \theta)$ and $\bar{c}_{2}(r, \theta)$ defined with respect to frame $\mathcal{A}$ appears:

$$
\begin{aligned}
& c_{1}(r, \phi)= \\
& =\underbrace{R_{l} \sin (k \theta)}_{:=\bar{c}_{1}(r, \theta)} \cos (k \chi)-\underbrace{R_{l} \cos (k \theta)}_{:=\bar{c}_{2}(r, \theta)} \sin (k \chi), \\
& =\bar{c}_{1}(r, \theta) \cos (k \chi)-\bar{c}_{2}(r, \theta) \sin (k \chi), \\
& c_{2}(r, \phi)=\bar{c}_{1}(r, \theta) \sin (k \chi)+\bar{c}_{2}(r, \theta) \cos (k \chi) \text {. }
\end{aligned}
$$

As a result of suitable transformations it may also be written:

$$
\begin{aligned}
& \bar{c}_{1}(r, \theta)=c_{2}(r, \phi) \sin (k \chi)+c_{1}(r, \phi) \cos (k \chi), \\
& \bar{c}_{2}(r, \theta)=c_{2}(r, \phi) \cos (k \chi)-c_{1}(r, \phi) \sin (k \chi) .
\end{aligned}
$$

The modes $\bar{c}_{1}(r, \theta)$ and $\bar{c}_{2}(r, \theta)$ are defined in the ALE-reference system $\mathcal{A}$ and are linear combinations of the modes $c_{1}(r, \phi)$ and $c_{2}(r, \phi)$ described in the Lagrangian frame $\mathcal{B}$, whereas the combination depends on $\chi$.

This information can be exploited in order to define a transformation: a deformation field resolved in the Lagrangian frame can be transformed to be resolved in the ALE frame and vice versa. Of course the physical deformation field itself does not change, but its resolution does so that the numerical values describing the deformation field will be different in frame $\mathcal{A}$ or $\mathcal{B}$, respectively.

In practise the transformation is formulated in terms of the modal amplitudes $q_{i}(t)$ which are the deformation variables in (11):

$$
\begin{aligned}
& \bar{q}_{i 1}(t)=\sin (k \chi(t)) \cdot q_{i 2}(t)+\cos (k \chi(t)) \cdot q_{i 1}(t), \\
& \bar{q}_{i 2}(t)=\cos (k \chi(t)) \cdot q_{i 2}(t)-\sin (k \chi(t)) \cdot q_{i 1}(t) .
\end{aligned}
$$

Again, the new modal amplitudes in the ALE frame $\bar{q}_{i}(t)$ are expressed as a linear combination of modal amplitudes in the Lagrangian frame $q_{i}(t)$ and it is just a matter of convenience and practicability in which coordinates the equations of motion are actually evaluated.

One particularity has been ignored so far. For mode shapes with $k=0$, i.e. no nodal diameters in Fig. 2, no mode couple with $c_{1}$ and $c_{2}$ according to (16) exists, since no associated sinus-function is introduced in (13). As a consequence the transformation (20) is not defined for such modes. However, eigenforms with $k=0$ present rotational-symmetric deformation fields since the dependency on $\phi$ is eliminated in (13) due to the term $\cos (k \phi)$. As a consequence eigenforms with $k=0$ are invariant with respect to rotations with angle $\chi$ or in other words: The modal coordinates $q_{i}(t)$ related to $k=0$ are identical in the ALE- and the Lagrangian description and no transformation is needed.

\section{The User Interface}

\subsection{Connectors and Parameters}

\section{annularPlate}

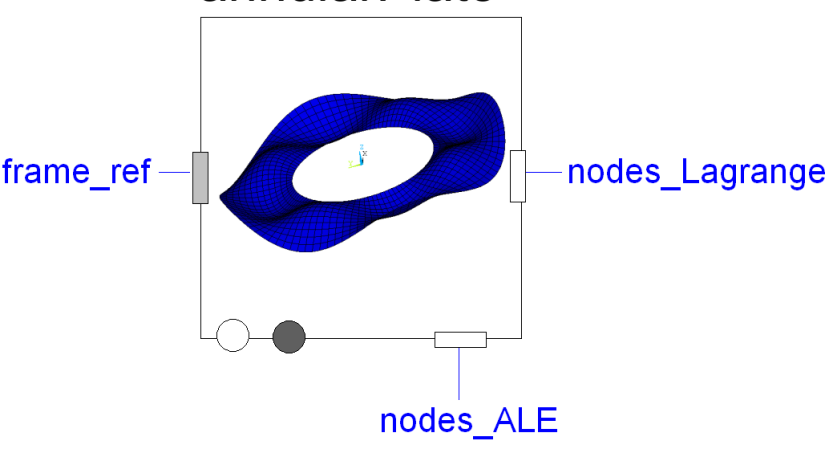

Figure 6: Icon layer of the AnnularPlate class with 3 types of multibody connectors: the floating frame of reference and two arrays of frames representing points in Lagrangian- or ALE-description, respectively.

Fig. 6 presents the AnnularPlate icon. Connections to the floating frame of reference of the plate are to be defined using the frame_ref connector. The array of connectors nodes_Lagrange contains as much frames as are given by the first dimension of the input parameter $x s i$ in the following table:

\begin{tabular}{lrl}
\hline \hline \multicolumn{3}{c}{ geometrical parameters } \\
\hline \hline$r_{-} i$ & {$[m]$} & inner radius of the plate \\
\hline$r_{-} a$ & {$[m]$} & outer radius of the plate \\
\hline$t h$ & {$[m]$} & thickness of the plate \\
\hline$x s i[:, 2]$ & {$[-]$} & points on the disc \\
\hline \hline
\end{tabular}

Each row of xsi specifies the radial and the angular position of one point in the mid-plane of the disc parametrized in the interval $[0,1]$, e.g. $x$ si $[1,:]=$ $\{0.5,0.125\}$ defines a point in the middle between the inner and outer radius at $45^{\circ}$ angular position.

The connector array nodes_ALE refers to the same input parameter definition $x s i$, whereas the associated points here are described in the ALE-representation. Forces and torques applied to these frames are in rest which respect to the rotation $\chi$ of the disc.

Note that conventional frame connectors from the Standard Multibody library are used within the Flexi- 
bleBodies library and no restrictions concerning connecting e.g. other bodies to nodes $A L E$ are effected, although nodes $\_$ALE frames do not represent material points.

Usually multibody frame connections represent physical mounting devices such as screws or welds that bond two frames together so that their positions and orientations are constrained to be identical. However it is the idea of nodes $A L E$ frames that they are not bonded to the disc and there is no mounting device. From the plate's material point of view nodes $A L E$ frames slide on the plate. In view of this fact the user is in charge to ensure that connections to nodes $A L E$ frames are physical consistent. If e.g. another body is attached to a nodes_ALE frame this would require a physical guidance device on the plate to which the external body is connected.

In addition to the 3-dimensional multibody frame connectors, two 1-dimensional rotational flanges are shown in Fig. 6. These two flanges are connected to both sides of the 3-dimensional rotational joint which is introduced into the AnnularPlate class at the plate axis by default. The two flanges are conditionally instantiated controlled via user parameter and can be utilized to e.g. define constant rotation velocity.

In addition to the purely geometrical parameters above, the table below shows the physical parameters the user has to provide in order to employ a AnnularPlate instance:

\begin{tabular}{lll}
\hline \hline \multicolumn{3}{c}{ physical parameters } \\
\hline \hline rho & {$\left[\mathrm{kg} / \mathrm{m}^{3}\right]$} & mass density \\
\hline$E$ & {$\left[\mathrm{~N} / \mathrm{m}^{2}\right]$} & Youngs's modulus \\
\hline$G$ & {$\left[\mathrm{~N} / \mathrm{m}^{2}\right]$} & Shear modulus \\
\hline
\end{tabular}

The following discretization parameters control the modal approach of the AnnularPlate model according to (13):

- boundaryConditionRI: This enumeration parameter offers the options free, supported and clamped and specifies the boundary condition at the inner radius.

- boundaryConditionRA: This is again an enumeration parameter that specifies the boundary condition at the outer radius in the same way as noted for the inner radius.

- nodalDiameters: This is an integer vector of arbitrary length, in which all nodal diameters numbers $k$, see Fig. 2, to consider have to be given. E.g. nodalDiameters $=\{0,2\}$ defines that all modes (to be additionally qualified by nodalCircles) with zero and two nodal diameters are to be taken into account.

- nodalCircles: This is an integer vector of arbitrary length, in which all nodal circles $l$ to consider have to be given, see Fig. 2.

- damping: This is a real vector, which defines the damping of each mode separately.

There is one aspect in which the discretization parameters above differ from what is depicted in (13). There, the number of mode shapes is specified by two thresholds $l_{m}$ and $k_{m}$ and all modes with $l \leq l_{m}$ and $k \leq k_{m}$ are included in the model. However the two inputs nodalCircles and nodalDiameters offer the possibility to specify each nodal circle and diameter to be considered, separately.

A literature review had revealed that in particular brake squeal models often only include a single mode shape couple corresponding to a specific frequency at which squeal phenomena have been observed in real applications, see e.g. [12]. The case is covered by the parameter definitions above.

Besides the discretization parameters that are related to the underlying plate model the graphical user interface of the AnnularPlate class consists of a bundle of other input data to specify in-scale and exaggerated animation, initialization, state selection and so on. Concerning these more general issues the user interface corresponds to what is already known from the Beam and ModalBody class of the DLR FlexibleBodies library.

\subsection{Degenerated Geometry}

There are two different cases of degenerated geometry which lead to singularities if defined by user input:

circular plate: The AnnularPlate model is not capable of representing a true circular plate with $r_{i}=0$. The model will simulate, if a very small inner radius such as e.g. $r_{i}=1^{-10} \mathrm{~m}$ is given, but as long as not enforced by clamped boundary conditions the displacement results on the inner radius do not satisfy the compatibility equations of continuum mechanics, see [10, Sec. II.6]. E.g. consider two displacements $\boldsymbol{u}_{A}\left(r_{i}\right)$ and $\boldsymbol{u}_{B}\left(r_{i}\right)$ of two arbitrary, but not coinciding points lying on the inner radius, then the following statement has to be noticed in general:

$$
\lim _{r_{i} \rightarrow 0}\left(\boldsymbol{u}_{A}\left(r_{i}\right)-\boldsymbol{u}_{B}\left(r_{i}\right)\right)^{2} \neq 0
$$


circular ring: It is also not possible to specify $r_{i}=r_{a}$. From the theoretical point of view the user may define an annular plate with arbitrary small width $r_{a}-r_{i}>0$, but as a consequence the eigen frequencies $\omega_{i}$ of the flexible body will be increased towards infinity:

$$
\lim _{r_{i} \rightarrow r_{a}} \omega_{i}=\infty
$$

\section{Example Models}

\subsection{A Lathe Cutting Model}

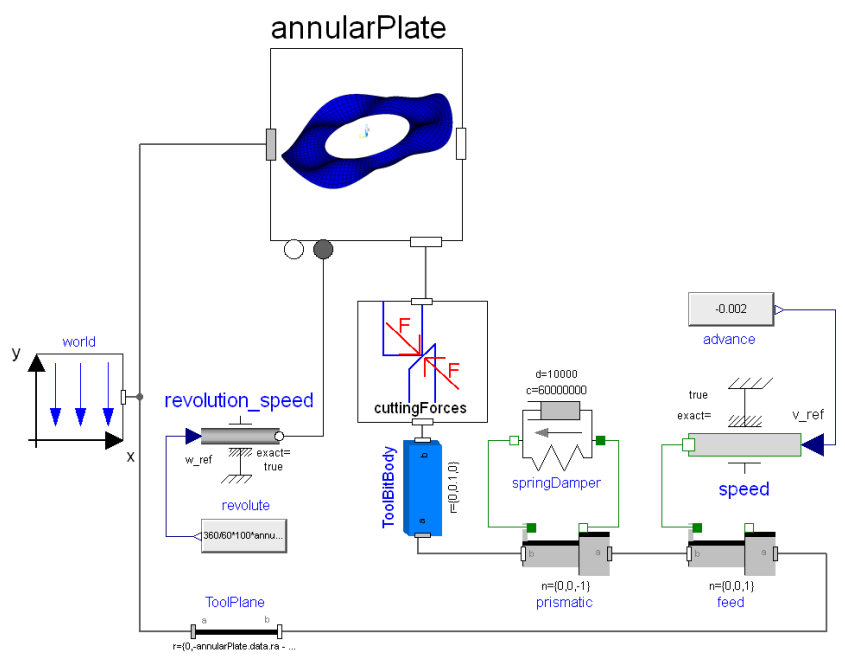

Figure 7: Diagram layer of the Lathe Cutting Model

The cylindrical turning of a disc on a lathe in Fig. 7 serves as an first example to demonstrate the approach. The disc, $r_{i}=0.075 \mathrm{~m}, r_{a}=0.15 \mathrm{~m}, t h=0.01 \mathrm{~m}$ made of steel, rotates with constant rotational velocity while the lathe tool is moved in parallel to the disc axis in order to form the outer cylindrical disc surface. The cutting tool is supported by a linear spring-damper element which represents compliances of the tool machinery.

The assumed cutting speed is $100 \mathrm{~m} / \mathrm{min}$, the feed is $120 \mathrm{~mm} / \mathrm{min}$. The relevant force here, the feed forward force $f_{f}$ is evaluated according to the instantaneous chip dimensions $b$ and $h$ :

$$
f_{f}=b h^{1-m_{f}} k_{f 1.1},
$$

using the specific force constants $m_{f}=0.7013, k_{f 1.1}=$ $351 \mathrm{~N} / \mathrm{mm}^{2}$.

The disc is assumed to be clamped at the inner radius and free at the outer radius.

Fig. 8 shows the animation of the simulation where the largest deformation is of course at the attachment point of the feed forward force.

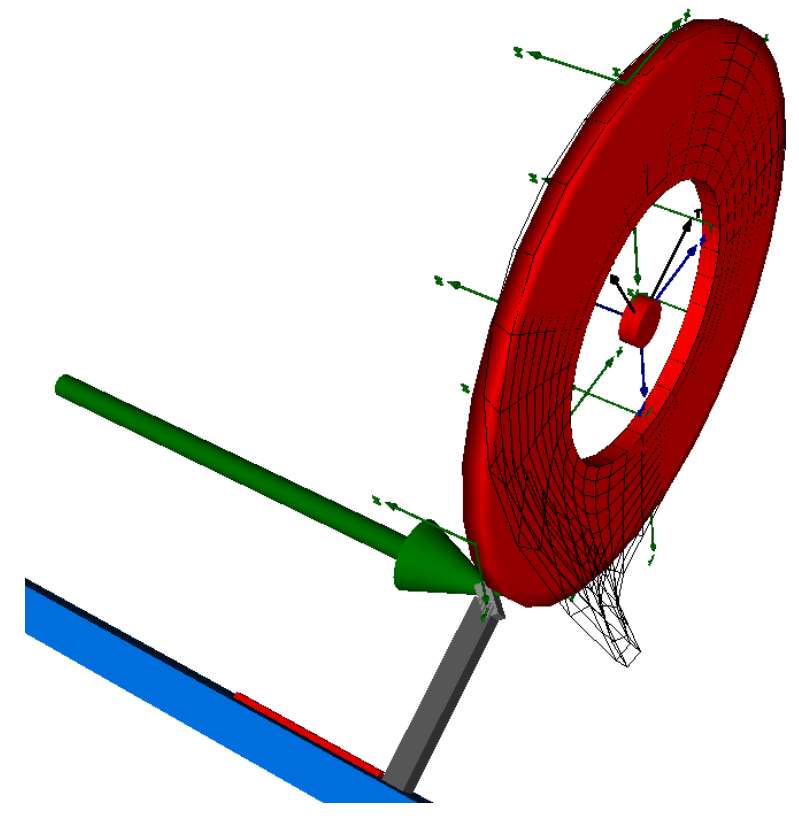

Figure 8: AnnularPlate model with applied nonrotating feed forward force, the solid animation shows the in-scale deformation at $t=0.9 \mathrm{~s}$, while the wireframe animation is exaggerated by a factor of 100 .

Fig. 9 depicts the time history of two deformations states $q_{i}(t)$ that are associated to the eigenforms with node diameter $k=1$ and node circle $l=0$. After $0.25 \mathrm{~s}$, the lathe tool approaches the plate and begins to cut. Then the chip dimensions are increased which leads to a larger feed forward force and larger deformations. After $1.23 \mathrm{~s}$ the cutting process is stationary.

The upper plot presents the Lagrange point of view, the virtual observer rotates with the plate and experiences how the deformations change with the rotation angle.

The lower plot delineates the standpoint of an observer that does not rotate with the plate. As a consequence the deformation of the plate is experienced to be stationary with respect to the rotation angle.

In order to verify the implementation the natural frequencies of the AnnularPlate model were compared to the results of a FEM analysis for different combinations of boundary conditions. Tab. 1 gives the results for the set-up used in the lathe cutting model. The differences are indeed very small for all boundary conditions so that at least the evaluation of the structural mass and stiffness matrices $\boldsymbol{M}_{e}$ and $\boldsymbol{K}_{e}$ can be assumed to be correct.

For two reasons this lathe cutting scenario is a challenging one: Firstly, the frequency of the excitation is much lower than the lowest natural frequency of the plate, i.e. this scenario is a quasi-static one. It 


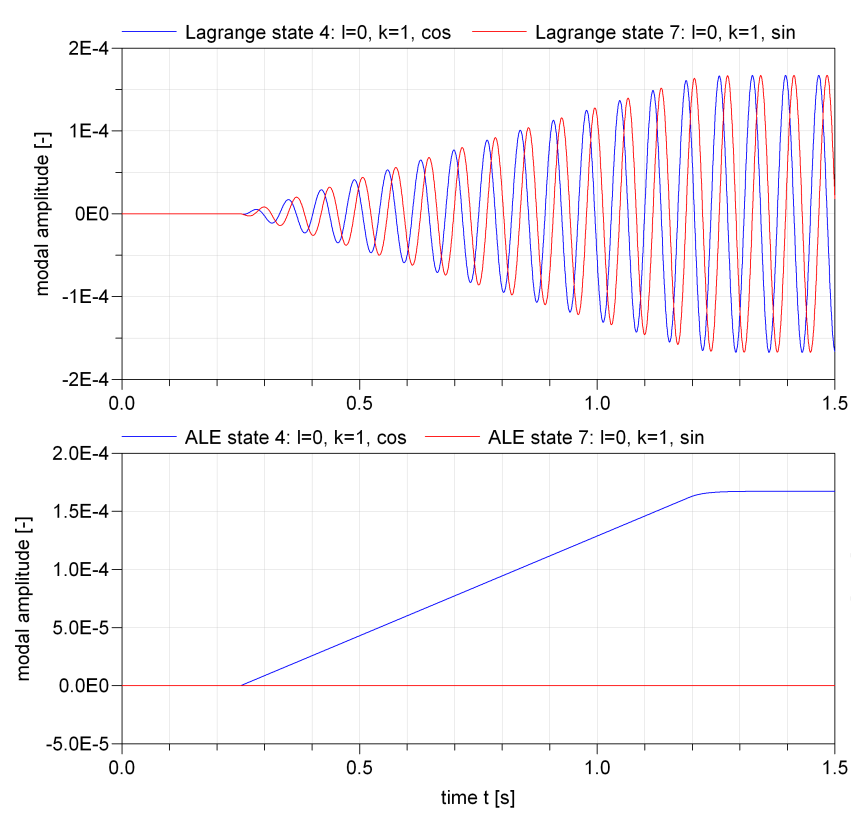

Figure 9: Modal amplitudes of two exemplary deformation states in Lagrangian and ALE-description.

is a known phenomenon that the discretization with eigenmodes is comparable inefficient whenever static deformation fields are to represent, so that a large number of modes is necessary to get correct values. Note that this fact does not apply for dynamic excitations.

Secondly, the application of a single, discrete force at the circumference of an annular plate is an issue for the angular discretization, which here may be interpreted as a Fourier expansion. Again, it is to expect that a large number of modes is necessary to get values close to reality.

A closer look at the exaggerated compared to the in-scale animation in Fig. 8 shows that deformations also occur in regions e.g. opposite to the force attachment point. These displacement results far away from the cutting tool are reduced if a higher number of nodal

\begin{tabular}{l|l|l|l|l|l}
\hline \hline \multicolumn{6}{c}{ Natural frequencies [Hz] } \\
\hline \hline \multicolumn{7}{c}{ Modelica } & 1449 & 1478 & 1478 & 1635 & 1635 \\
\hline Ansys & 1451 & 1480 & 1480 & 1637 & 1637 \\
\hline \hline \multicolumn{7}{|c}{} \\
\hline Modelica & 2064 & 2064 & 2847 & 2847 & 3974 \\
\hline Ansys & 2065 & 2065 & 2848 & 2848 & 3977 \\
\hline \hline
\end{tabular}

Table 1: The first 10 natural frequencies of an AnnularPlate model compared to an Ansys model of the same plate with 1296 Shell63 elements for the sake of verification. diameters $k$ is considered.

The convergence of the deformation results as a function of the nodal diameters $k$ and the nodal circles $l$ is presented in Fig. 10, where the deformation at the force attachment points are given. At least the nodal

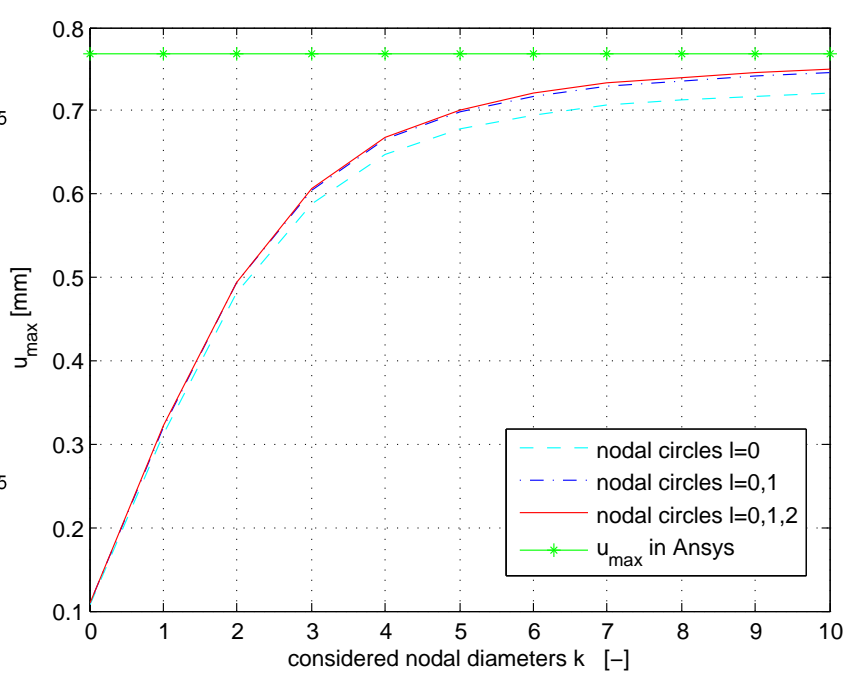

Figure 10: Convergence of the displacements results at the force attachment point

diameters $k=0,1, \ldots, 7$ and the nodal circles $l=0,1$ should be taken into account in order to get reasonable static deformation results which leads to all together 30 degrees of freedom. The highest frequency in the model associated to the eigenform with $k=7, l=1$ turns out to be $18837 \mathrm{~Hz}$. In the first implementation $44.7 \mathrm{cpu}-\mathrm{s}$ were required to simulate the $5.5 \mathrm{~s}$ of the complete scenario with a common lap-top.

\subsection{A Helicopter Blade Control Model}

The cyclic blade control of an helicopter is the second application example shown in Fig. 11. The swash plate, here modeled as an annular plate, supports two linkages that actuate the pitch joint of the helicopter blades. As long as the swash plate rotates in parallel to the rotor base carrying the blades above, the pitch of the blades is kept constant during one rotation. If the swash plate is tilted in such a way that the angular velocity vectors of the rotor base and the swash plate are no more collinear, the blade pitches are a function of the rotation angle, see Fig. 12.

Since the direction and value of the air forces acting on the blades depend on the pitch angle, the roll and pitch motion of the helicopter fuselage can be controlled via this mechanism.

Besides the swash plate, the linkages, the rotor base and the pitch joints, the model in Fig. 13 contains 


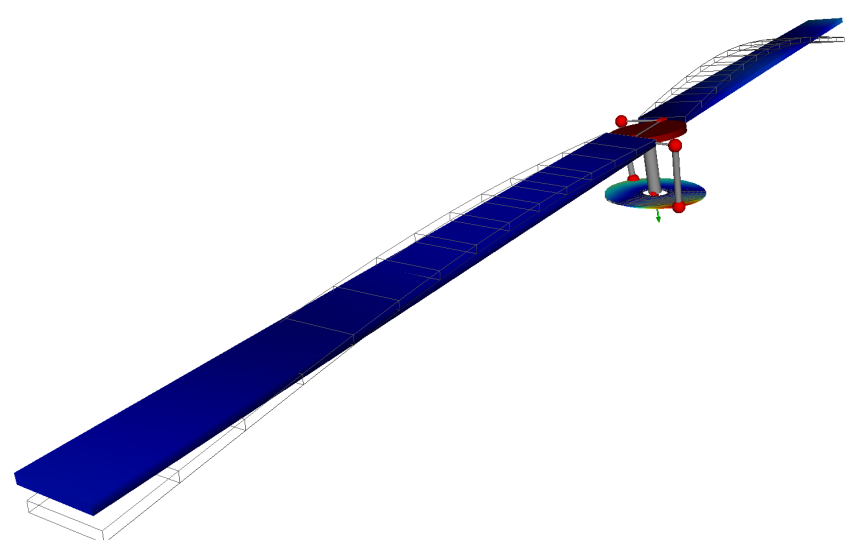

Figure 11: Total view on the helicopter mechanism: the wireframe illustrations exaggerate the deformations by a factor of 100 , while the solid representations are shown in true scale.

two $5 \mathrm{~m}$ long beams describing the blades and considering their torsional and two-directional bending deformation. A rough representation of the air forces' effects on the pitch motion is given by force-damper elements acting on the pitch joints. The prismatic joint in Fig. 13 allows for the adjustment of the vertical position of the swash plate and thereby governs the collective pitch angle. The rotational joint aside regulates the tilting angle of the swash plate and therefore provides cyclic blade control.

The inner and outer radius of the $0.01 \mathrm{~m}$ thick swash plate made of steel is set to 0.1 and $0.39 \mathrm{~m}$, respectively. Supported boundary conditions are applied at the inner radius and the $l=0, k=0,1, \ldots, 7$ eigenforms are considered, so that 15 degrees of freedom and eigenfrequencies between $50 \mathrm{~Hz}$ and $1009 \mathrm{~Hz}$ are defined. Since the externals loads acting on the plate here rotate with the plate the ALE-functionality was

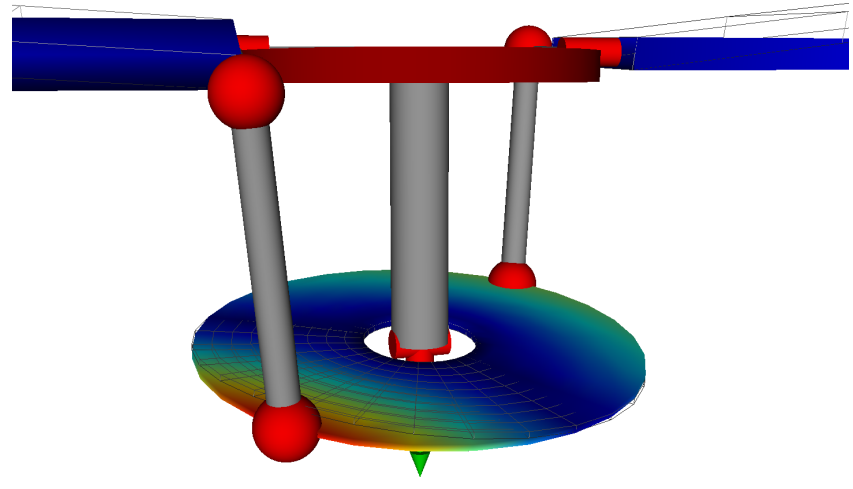

Figure 12: Side View on the tilted Swash Plate: the absolute value of the deformations are additionally indicated by color.

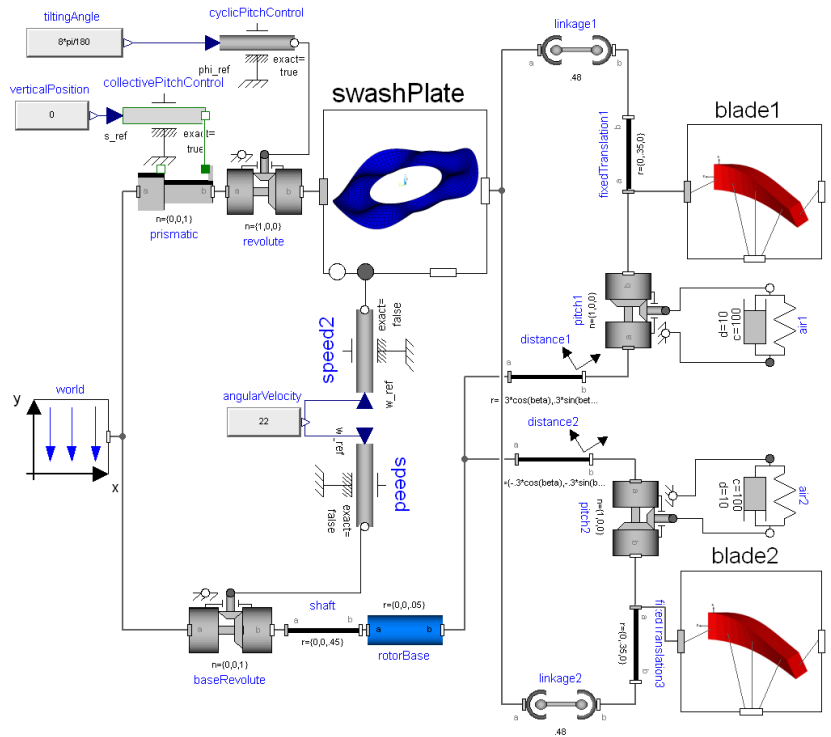

Figure 13: Diagram layer of a Helicopter Blade Control Model

not used. The blade models take the first eigenform for each of the three deformations types into account. In order to evaluate $1 \mathrm{~s}$ simulation time, $4.7 \mathrm{cpu}-\mathrm{s}$ are required on a common lap-top.

The simulation scenario assumes a constant angular motion of the blades with $22 \mathrm{rad} / \mathrm{s}$, the swash plate tilting angle is as well constant, namely $8^{\circ}$. Fig. 14 shows the controlled pitch angles as a function of time.

The above plot in Fig. 15 presents the bending deformation of the plate at those two positions where the linkages are attached to. Since the model is initialized in the undeformed configuration, natural vibration are initiated but are damped out rather quickly due to the defined structural damping of $2 \%$.

The first modal amplitude in the plot below in Fig. 15 is associated to the rotational-symmetric $<k=$ $0, l=0>$-eigenform and its stationary value $q_{1} \neq 0$ is ruled by the gravity load. The modal amplitude $q_{2}$

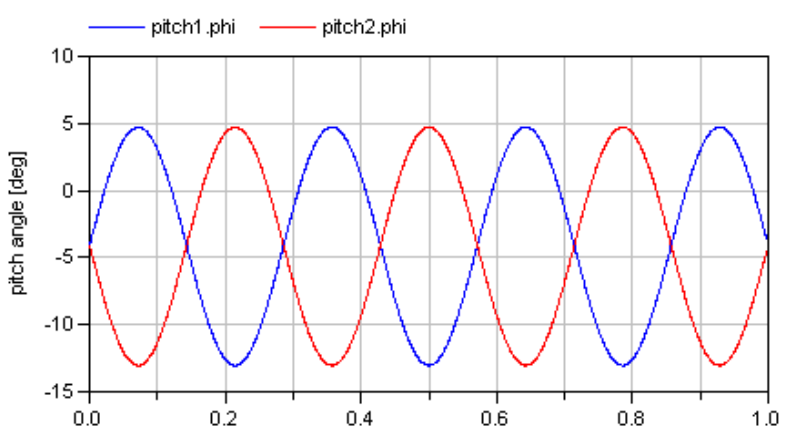

Figure 14: Simulation results concerning the controlled helicopter blade pitches. 

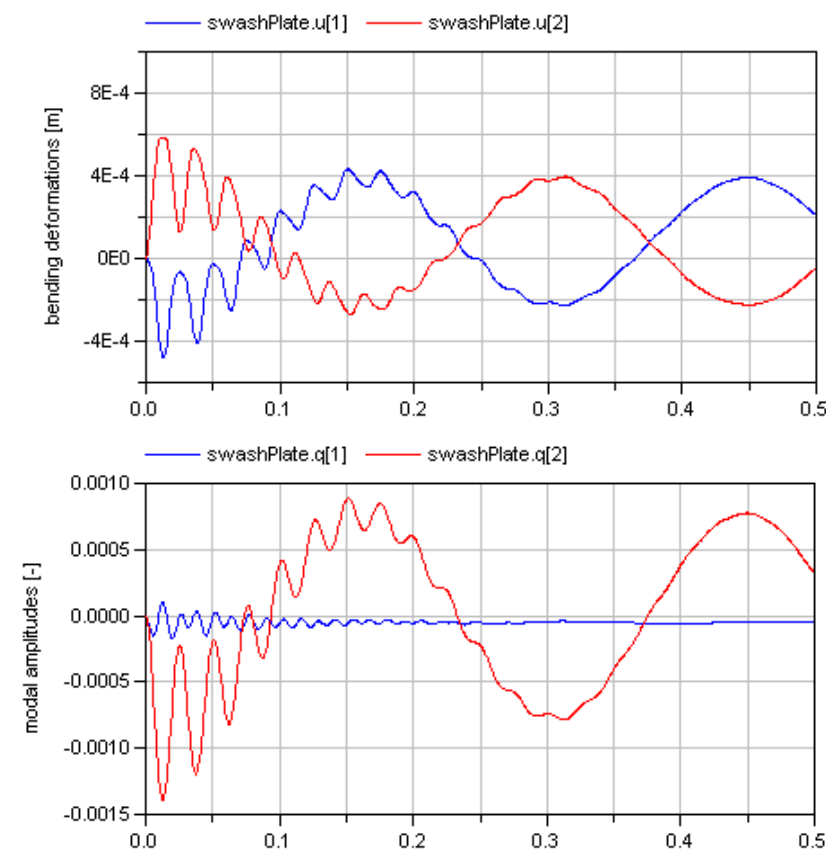

Figure 15: Deformation results at the two linkage attachment points and two modal amplitudes of the swash plate.

is related to that $<k=1, l=0>$-eigenform, which displays its maximum and minimum deformations exactly at the linkage attachment points. As a consequence $q_{2}$ represents by far the dominating part of the particular solution.

\subsection{A Brake Squeal Model}

The last application is a reproduction of a brake squeal model presented by Chakraborty et.al. [12]. It is based on the idea that the friction forces are oriented along

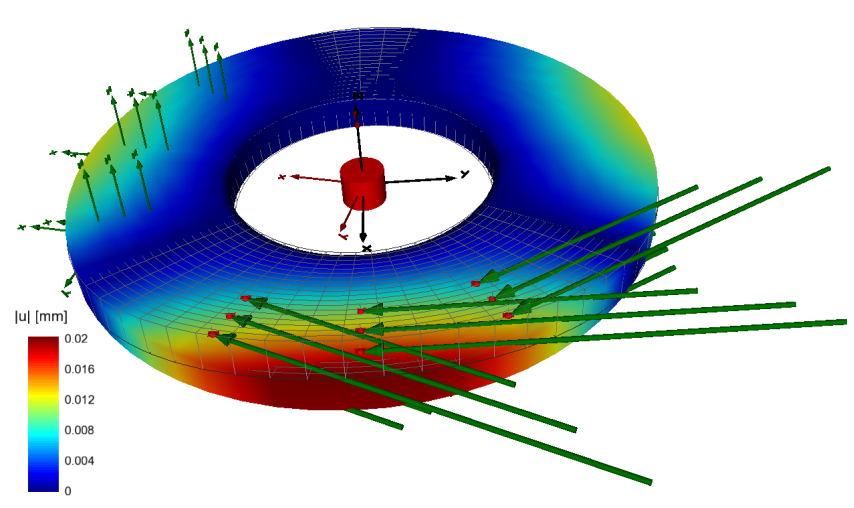

Figure 16: Animation of the brake disc with 18 applied friction forces oriented along the deformed surface (wireframe scale 1000:1). Pads and caliper bodies are considered but omitted for the visualization only.
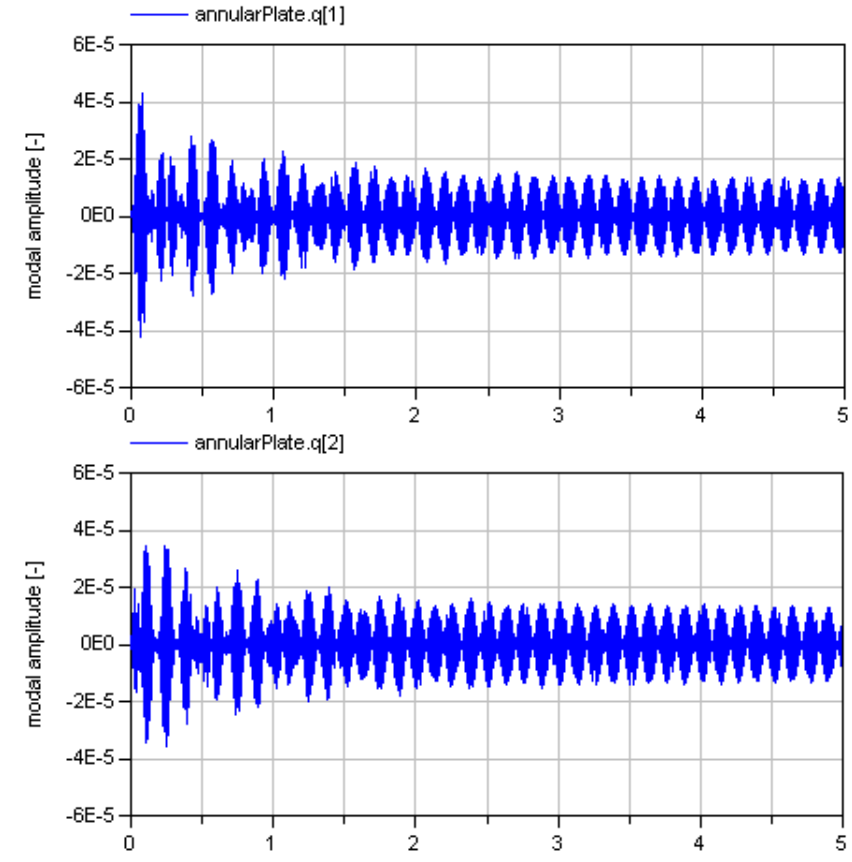

Figure 17: Modal amplitudes of the Brake Squeal Model.

the deformed friction surface. This so-called followerforces phenomenon leads to a flutter-type instability and as a consequence to brake squeal. The arrows in the animation Fig. 16 show the friction forces as they are aligned with the surface tangent at the contact points. Due to this set-up the limit cycles in Fig. 17 occur as soon as the friction coefficient exceeds a certain limit.

The simulation scenario was defined as an initial value problem. Therefore the modal amplitudes of the first $1.5 \mathrm{~s}$ in Fig. 17 slightly differ from the behavior later on. The angular velocity of the brake disc was assumed to be constant $25 \mathrm{rad} / \mathrm{s}$, the brake disc dimensions were set to $r_{i}=0.07 \mathrm{~m}, r_{a}=0.153 \mathrm{~m}$ and $t h=0.0181 \mathrm{~m}$ and 4 eigenforms with $l=0, k=1,2$ with supported boundary conditions at the inner radius are considered. $64 \mathrm{cpu}$-s were needed to simulate the $5 \mathrm{~s}$ to be seen in Fig. 17.

The contact is formulated with one prismatic joint in axial direction for each contact point, see Fig. 18. The spherical joint allows for the alignment of the friction force with the contact surface. frame_b is to be connected to one nodes_ALE frame of the annular plate, see Fig. 6. frame_a is supposed to provide the connection to the brake caliper, which is a part of the model but not visualized in Fig. 16. The springdamper element attached to the prismatic joint represents the contact stiffness. For a more advanced study, this linear element may be replaced by a non- 


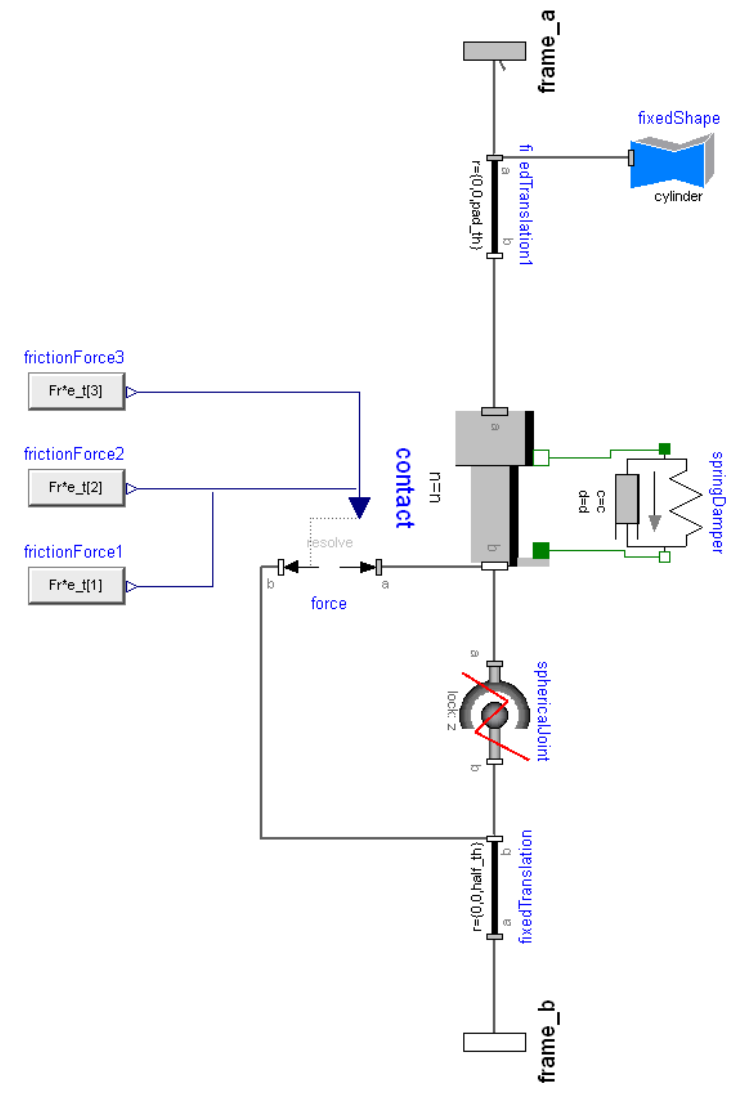

Figure 18: Diagram layer of the contact submodel.

linear spring which takes the loss of contact or the liftoff of the brake pads, respectively, into account. The simplicity of the contact modeling here again demonstrates the advantages of the ALE-description.

\section{Conclusions and Outlook}

This paper introduces the new Modelica class called AnnularPlate. The underlying plate model refers to a homogeneous Kirchhoff plate in cylindrical coordinates. The option to use connector frames in the socalled Arbitrary Lagrangian-Eulerian description offers the capability to apply non-rotating external loads in a convenient and numerical efficient way. The first example, a lathe cutting model, demonstrates in particular the advantages of this ALE-approach. The Helicopter Blade Control model presents the annular plate model as a part of a more complex mechanism. A Brake Squeal Model from literature concludes the example presentation. The AnnularPlate class will be distributed with the Version 2.0 of the commercial DLR FlexibleBodies library.

Future enhancements concern the second order displacement field description to cover initial plate buckling phenomena as well. The additional consider- ation of torsional deformations of the plate is another optional improvement in order to cope with applications in which large forces in circumferential direction are present.

\section{Acknowledgements}

A first preliminary version of the annular plate model was implemented by Kemal Çiğ in the course of his master thesis project at the DLR.

This work is part of the ITEA $2 \sim 6020$ project Eurosyslib and therefore funded by the German Federal Ministry of Economics and Technology. The authors highly appreciate the partial financial support of DLR by BMBF (BMBF Förderkennzeichen: 01IS07022F), the German Federal Ministry of the Education and Research, within the ITEA 2 project Eurosyslib [13].

\section{References}

[1] Andreas Heckmann, Martin Otter, Stefan Dietz, and José Díaz López. The DLR FlexibleBody library to model large motions of beams and of flexible bodies exported from finite element programs. In 5th International Modelica Conference, pages $85-95,2006$.

[2] O. Wallrapp. Standardization of flexible body modeling in multibody system codes, Part 1: Definition of standard input data. Mechanics of Structures and Machines, 22(3):283-304, 1994.

[3] A.W. Leissa. Vibration of plates. NASA SP-160, Washington, D.C., 1969.

[4] R. Szilard. Theory and Analysis of Plates. Prentice-Hall, Inc., Englewood Cliffs, New Jersey, 1974.

[5] H. Irretier. Grundlagen der Schwingungstechnik 2. Vieweg-Verlag, Braunschweig, 2001.

[6] O. Wallrapp and R. Schwertassek. Representation of geometric stiffening in multibody system simulation. International Journal for Numerical Methods in Engineering, 32:1833-1850, 1991.

[7] F. Bloom and D. Coffin. Handbook of Thin Plate Buckling and Postbuckling. Chapman \& Hall/CRC, Washington, D.C., 2001. 
[8] A.A. Shabana. Dynamics of Multibody Systems. Cambridge University Press, Cambridge, 2nd edition, 1998.

[9] Carl de Boor. A practical Guide to Splines. Springer-Verlag, Berlin, 1978.

[10] J. Salençon. Handbook of Continuum Mechanics. Springer-Verlag, Berlin, 2001.

[11] U. Nackenhorst. The ALE-formulation of bodies in rolling contact, Theoretical foundations and finite element approach. Computer Methods in Applied Mechanics and Engineering, 193:42994322, 2004.

[12] G. Chakraborty, T. Jearsiripongkul, U. von Wagner, and P. Hagedorn. A new model for a floating caliper disc-brake and active squeal control. VDI-Bericht, 1736:93-102, 2002.

[13] ITEA 2. Eurosyslib. http://www.itea2.org. 